\title{
Coulisses
}

Revue de théâtre

42 | Printemps 2011

Racine : Théâtre et émotion

\section{La mise en scène du tragique racinien dans les éditions illustrées}

Marie-Claire Planche

\section{OpenEdition}

1 Journals

Édition électronique

URL : https://journals.openedition.org/coulisses/688

DOI : $10.4000 /$ coulisses.688

ISSN : 2546-9460

Éditeur

Presses universitaires de Franche-Comté

Édition imprimée

Date de publication : 15 février 2011

Pagination : 69-80

ISBN : 978-2-84867-316-5

ISSN : 1150-594X

Référence électronique

Marie-Claire Planche, "La mise en scène du tragique racinien dans les éditions illustrées », Coulisses

[En ligne], 42 | Printemps 2011, mis en ligne le 30 novembre 2016, consulté le 29 décembre 2022.

URL : http://journals.openedition.org/coulisses/688; DOI : https://doi.org/10.4000/coulisses.688 


\title{
La mise en scène du tragique racinien dans les éditions illustrées
}

\author{
Marie-Claire Planche \\ Université de Bourgogne
}

Les pièces de Jean Racine ont toutes été illustrées, et ce du vivant de l'auteur, puisque la première édition collective a paru en 1676. Les trois pièces qui nous intéressent ici, La Thébaïde ou les Frères ennemis, Britannicus et Mithridate ont été représentées respectivement en 1664, 1669 et 1673. Il paraît utile de situer ces pièces dans la carrière du poète : La Thébaïde est sa première tragédie, Britannicus succède aux Plaideurs (sa seule comédie), tandis que Mithridate vient juste avant les deux dernières tragédies mythologiques : Iphigénie (1674) et Phèdre (1677). Parmi les nombreuses illustrations qui accompagnent les éditions des pièces du dramaturge, nous avons dû faire un choix, dont nous espérons qu'il permettra de cerner les enjeux de la transposition d'épisodes raciniens dans les arts visuels. Nous avons retenu deux estampes pour chacune de ces pièces, toutes issues d'éditions du XVIII ${ }^{\mathrm{e}}$ siècle, dont l'iconographie est certainement moins connue que celle du XVII ${ }^{\mathrm{e}}$ siècle. Les éditions des Euvres de Racine, au XVIII ${ }^{e}$ siècle, célèbrent le poète qui bénéficie de huit éditions illustrées.

Il fallait cependant trouver une unité à ce corpus, ce que les estampes de l'édition de 1760 dessinées par Jacques De Sève, proposent. En effet, la qualité et l'animation des vignettes inventées par cet artiste attestent de la réception des tragédies de Racine et du soin avec lequel ses pièces ont été illustrées. Dans le livre, les estampes sont toujours placées en position liminaire, avant le texte de chaque pièce. Elles satisfont ainsi l'œil du lecteur et représentent le plus souvent un instant éminemment tragique. Ce principe, qui peut rappeler que les tragédies sont destinées à être représentées, montre aussi son autonomie par rapport à la scène théâtrale. En effet, les épisodes retenus par les dessinateurs ne sont pas tous joués puisque certains de ces événements sont connus dans les tragédies par un récit, vivant et animé. Les dessinateurs, qui n’obéissaient pas 
aux contraintes de la scène théâtrale, ont fréquemment choisi les drames connus par le seul récit : les illustrations des différentes éditions des CEuvres de Racine, publiées de 1676 à 1801 en témoignent. Aussi avons-nous souhaité nous intéresser à des estampes représentatives de ce principe. Il nous semble maintenant utile de présenter le corpus sur lequel s'appuie notre propos. Les deux vignettes de La Thébaïde illustrent dans un décor naturel le combat des frères ennemis, Étéocle et Polynice, qui s'affrontent au pied des murailles de Thèbes. La première estampe est issue de l'édition de 1760 marquée par le goût rocaille [fig. 1], tandis que la seconde, dessinée par Jean-Jacques-François Le Barbier pour l'édition de 1796 s'inscrit dans le courant néoclassique [fig. 2]. Deux récits majeurs rythment Britannicus : le rapt de Junie par Néron (I, 3) et la mort de Britannicus au cours du banquet de la réconciliation $(V, 5)$. Cette scène, qui marque la fin de la tragédie, est relatée avec finesse par Burrhus. L'instant fatal fut l'objet d'illustrations variées, comme le montrent les vignettes de l'édition de 1723 et de 1760 [fig. 3 et 4]. Enfin, pour Mitbridate, nous avons choisi non seulement l'épisode de la mort du roi de Pont relaté par Arbate $(\mathrm{V}, 4)$ et par une scène dialoguée [fig. 6], mais aussi un instant joué sur la scène et dans lequel la mort d'une femme, Monime, est, semble-t-il, imminente [fig. 5]. Nous ne pouvons pas, dans le cadre de ce travail définir tous les aspects d'une transposition dans les arts visuels, mais espérons pouvoir présenter, à travers ces exemples, quelques caractéristiques de l'iconographie racinienne ${ }^{1}$.

Racine, dans ses pièces, a proposé des récits qui sont comme autant de tableaux dans lesquels le sens du drame est mis en œuvre à l'aide d'une palette d'émotions et de couleurs. Le dramaturge a, nous le pensons, écrit ses pièces comme un peintre compose son tableau, un tableau dans lequel se joue le drame, tout entier contenu sur une toile unique qui permet de disposer les personnages. L'ordonnancement est rigoureux et pour chaque pièce, le lieu de l'action est campé par des noms dont la puissance évocatrice laisse place à l'imaginaire. Puis les dialogues font surgir des personnages animés de fortes passions qui s'impriment sur leurs corps. Les agitations de l'âme, les passions qui modèlent les héros sont alors exposées. Ainsi façonnés, ils s'animent et disent quelques-unes de leurs postures ou de leurs déplacements. Enfin, pour achever sa composition, le dramaturge distribue ombre et lumière, révélant la noirceur ou l'éclat des cœurs. Parfois le récit, tableau dans le tableau, s'immisce dans l'histoire qui a été peinte. Le procédé est proche de celui que nous venons de décrire, laissant une place remarquable à la couleur et au mouvement. Après avoir pris soin d'indiquer le point de vue, celui du narrateur-témoin, Racine a brossé un tableau d'ensemble dans lequel il livre les tonalités, puis s'attache aux attitudes des personnages et aux éléments du décor, pour enfin laisser entendre

1. Nous renvoyons le lecteur à notre ouvrage : De l'iconographie racinienne, dessiner et peindre les passions. Turnhout, Brepols, 2010. 
le bruit et les cris. Les passions dont Racine anime ses héros et les différents registres expressifs sont la manifestation d'une grande maitrise. Le dramaturge manie les outils du peintre et connait la science de l'orateur. Il a retenu les leçons de Cicéron et de Quintilien et participe de ce grand élan commun aux hommes du XVII siècle en faveur des passions.

Le récit s'impose sur la scène théâtrale, il répond, comme Boileau l'a expliqué, à une nécessité. En s'inspirant d'Horace, il l'a formulé dans une définition justifiant le recours à l'hypotypose, qui est « la représentation des faits proposée en termes si expressifs que l'on croit plutôt voir qu'entendre $»^{2}$.

Ce qu'on ne doit point voir, qu'un récit nous l'expose

Les yeux en le voyant saisiraient mieux la chose ;

Mais il est des objets que l'art judicieux

Doit offrir à l'oreille et reculer des yeux 3 .

Les récits raciniens usent de cette figure rhétorique avec une belle adresse qui contribue à la forte impression qu'ils suscitent. Cette figure stylistique est descriptive au théâtre ou en rhétorique, elle frappe les esprits et suggère une image ${ }^{4}$. Les préceptes de Boileau s'inscrivent dans un contexte de réflexion sur l'art théâtral. En effet, à partir de 1640, la bienséance dicte ses règles, interdisant la représentation sur scène de combats, de meurtres ou de lascivités. L'empoisonnement de Britannicus, les suicides d'Hermione ou d'Ériphile, la mort sanglante d'Hippolyte se déroulent donc en coulisses. La relation donne des indications sur les réactions des personnes présentes, tout en restituant l'animation de la scène. Ainsi Burrhus décrit-il les sentiments partagés de la foule, l'étonnement de Narcisse ou la compassion feinte de Néron après la fulgurance de la mort de Britannicus. Le narrateur suscite chez ceux qui l'écoutent une image mentale qui cristallise souvent l'un des épisodes les plus dramatiques. Le ton est parfois très vif, les vers incisifs et sans détour.

2. Quintilien, Institution oratoire, Paris, Belles Lettres, 1975, établie par Jean Cousin, IX, 2, 40. Pierre Fontanier, Les Figures du discours. Paris, Flammarion, 1977 a repris et développé cette définition dans des termes qui conviennent bien à notre propos: «L'hypotypose peint les choses d'une manière si vive et si énergique qu'elle les met en quelque sorte sous les yeux, et fait d'un récit ou d'une description, une image, un tableau ou même une scène vivante ", p. 390 .

3. Boileau, Art poétique, 1674. CEurres, Paris, Garnier-Flammarion, 1969, établie par Sylvain Menant, chant III, v. 51-54.

4. Marc Fumaroli, Héros et orateurs. Rhétorique et dramaturgie cornéliennes, 1990, Genève, Droz, 1995: «Autres figures à la disposition de l'orateur pour graver dans l'imagination de l'auditoire : l'hypotypose, ou demonstratio, évocation hallucinatoire d'un lieu ou d'une scène reconstituée vraisemblablement, comme un tableau vivant enchâssé dans le discours [...] Et toutes ces évocations, qui s'adressent aux sens intérieurs, frappés par la magie du verbe et du verbe seul, accompagné par l'actio, doivent être faites avec la plus grande force d'évidence, comme des apparitions magiques, luttant victorieusement avec les données immédiates des sens extérieurs », p. 297. 
Quant au temps du récit, qui n'est pas celui de l'ensemble de la pièce, il acquiert une autonomie, non seulement parce que l'événement rapporté s'est déjà déroulé, mais aussi par le rythme donné à la relation. L'attention de l'auditoire est retenue par la succession des faits, elle l'est aussi par la force évocatrice des images que le narrateur fait surgir: le récit participe ainsi pleinement de la catharsis. Comme dans les arts visuels, il y a dans le même temps une volonté d'impliquer celui qui écoute jusqu'à faire de lui un spectateur, et une mise à distance par la forme inhérente au récit ${ }^{5}$. Ces narrations sont comparables à des tableaux qui exposent avec cohérence et vie un événement, dans une unité de ton et de coloris particulièrement efficace. Les récits soulignent l'enchainement des événements qui conduisent jusqu'à l'instant final, ménageant des pauses que les artistes ont su transposer à plusieurs reprises. L'un des pouvoirs de la poésie et de la peinture est de figurer des scènes insoutenables dans la réalité qui, une fois composées, peuvent séduire le lecteur et le spectateur. La compositio et la mise à distance qui procèdent des arts d'imitation sont les conditions indispensables de la réception par le public. La transposition d'un sujet littéraire dans les arts visuels peut également prendre la forme d'un commentaire si l'on considère la part d'interprétation dévolue à l'artiste. Les thèmes raciniens n'ont pas dérogé à ces principes ; les différentes représentations qui en ont été données dans la peinture et la gravure restituent ce processus tout en soulignant l'extraordinaire fécondité des pièces. Le souhait de proposer aux spectateurs ou aux lecteurs des compositions représentatives s'exprime d'une manière prégnante. Les illustrations, quel qu'en soit le support offrent des pérégrinations dans les textes et dans le temps, restituant tout un monde sensible. Les réflexions théoriques, les moyens mis en œuvres par les artistes parlent d'une même voix pour célébrer les possibilités respectives de la peinture et de poésie, tout en rappelant leurs convergences.

Les combats et les luttes qui se déroulent en coulisses ont été à l'origine de plusieurs morts dans les tragédies raciniennes. Le mouvement, le rythme que nous venons d'évoquer se lit dans le combat acharné que se livrent les frères ennemis de La Thébaïde. Ainsi le duel sanglant d'Étéocle et de Polynice au cours duquel Hémon a aussi perdu la vie a-t-il été représenté dès l'édition de 1676. C'est près de la ville de Thèbes, en dehors de ses murailles que se déroule le combat des deux frères; le lieu du duel est situé avec précision par Créon :

Ils ont choisi d'abord pour leur champ de bataille,

Un lieu près des deux camps, au pied de la muraille ${ }^{6}$.

Un peu plus avant dans le récit, il évoque les fortifications de la ville en livrant les réactions de la foule qui se tient « sur le haut des tours $»^{7}$. La scène se dé-

5. Marc Bayard, «Intermédiaires et admoniteurs dans l’art théâtral et pictural », Marc Bayard, dir., L'Histoire de l'art et le comparatisme. Paris, Somogy, 2007, p. 53-69.

6. La Thébaïde, V, 3, v. 1317-1318.

7. Id., v. 1357. 
roule donc à l'extérieur et les dessinateurs ont délimité l'espace par une lice derrière laquelle la foule des soldats se masse, relayée par celle qui regarde le duel d'un point de vue élevé. Certains sont sur un promontoire naturel, tandis que d'autres sont sur les créneaux des tours et des murs de la ville. Dans la vignette de De Sève [fig. 1], les bâtiments de Thèbes se dressent au-dessus de l'enceinte ; leurs formes carrées ou circulaires alternent dans un ensemble architectural assez dense et parfaitement fantaisiste. La composition de François Chauveau pour l'édition de 1676 a constitué un véritable modèle pour les vignettistes des éditions postérieures qui se sont largement inspirés de la disposition des lieux. Si les dessinateurs ont modulé le sujet, si leur vision de la ville a revêtu des formes diverses, ils ont cependant tous laissé l'espace largement ouvert. La hiérarchisation des plans et la disposition étaient en effet propices à la représentation d'une foule nombreuse, répartie en différents points de la composition. Le récit de Créon, plutôt long, ménage des pauses et n’est pas exempt de rebondissements. Il offre ainsi aux dessinateurs, la possibilité de représenter différents instants de la lutte. Ce combat, annoncé dès le début de la pièce, mis en scène par les références au décor de la ville, est d'une extrême violence que Créon a fidèlement relatée.

Jacques De Sève a choisi pour le frontispice le début du combat [fig. 1]. Avec l'énergie qui caractérise ses vignettes, il a proposé un duel très enlevé : les gestes souples des deux frères sont emplis de force. Les étoffes volent, les corps sont en équilibre, les poings serrés, le regard de Polynice est perçant, aussi aiguisé que l'arme qu'il s'apprête à enfoncer dans le corps de son frère. L'équilibre précaire d'Étéocle à terre, est, au-delà de l'instant de lutte représenté, porteur de sens puisque cet instant précède le retournement de situation. C'est avec une rare violence que Polynice se jette sur Étéocle qui tente de résister en parant le coup de son bouclier. La nature des sentiments qui animent ces frères ennemis est exposée avec une grande vérité : il s'agit d'une lutte et non de poses destinées à suggérer le combat. Les spectateurs du duel jouent un rôle essentiel dans la représentation des émotions puisque leurs attitudes sont expressives. Les soldats du premier plan sont attentifs et craintifs, tandis que la foule, extrêmement nombreuse, est animée, en mouvement. Le dessinateur n'a pas négligé le rôle des personnages secondaires qui participent pleinement de l'expressivité de la scène. La gestuelle, les visages sont autant d'indices d'une foule qui a crié sa peine ou son empathie. Ces personnages ont été en outre habilement disposés, sur divers plans variés et jusque sur des points particulièrement élevés. Enfin, la place du spectateur de l'estampe a été définie : elle se trouve au tout premier plan, devant la brèche ouverte dans la lice permettant un accès privilégié à la scène principale. La proximité avec les soldats qui se tiennent à droite et ne cachent pas leurs sentiments rappelle que la composition est destinée à susciter des émotions. Mais le récit de Créon ne s'arrête pas à cet épisode, le combat n’est pas terminé. Alors que Jacques De Sève s'est intéressé 
à la première partie du récit, Jean-Jacques François Le Barbier a, quant à lui, choisi d'illustrer le revirement de situation. Étéocle, laissé pour mort, n’est que blessé lorsque Polynice vient le désarmer [fig. 2] :

Et dans l'instant fatal que ce frère inhumain

Lui veut ôter le fer qu'il tenait à la main,

Il lui perce le cœur; et son âme ravie,

En achevant ce coup abandonne la vie ${ }^{8}$.

Le mouvement d'Étéocle est soudain, il se redresse vivement pour porter l'estocade. Dans cette estampe, l'effet de surprise est préservé, et le corps sans vie d'Hémon au second plan indique qu'il s'agit de la seconde phase de l'épisode, comme le rappellent les vers gravés qui succèdent à ceux que nous avons cités. La mise en scène éloigne la foule dont les silhouettes ne sont qu'esquissées, limitant la valeur expressive des spectateurs. La représentation de la mort prochaine n'est pas dénuée d'accents théâtraux, mais il y a dans ce duel une forme de froideur qui limite l'émotion du spectateur. Ces deux estampes permettent de comprendre quelques enjeux de la transposition d'un récit dans les arts visuels et de souligner les points communs unissant le texte et l'illustration. La vivacité et la violence du combat que le récit de Créon a su mettre en œuvre, les réactions vibrantes de la foule ont été transposées avec suffisamment d'efficacité pour que ces estampes acquièrent un statut autonome.

Racine fait voyager son public de Thèbes aux rives du Bosphore, ouvrant ainsi les murailles sur la mer dont le rôle dans les tragédies n'est pas négligeable. Les personnages embarquent ou débarquent, Enone se précipite dans les flots d'où le monstre a surgi ; et l'absence de vent marin est, quant à lui, à l'origine du drame d'Iphigénie. Cependant, le port qui est le lieu de l'action de Mitbridate, n'apparait pas toujours dans les illustrations des différentes éditions. Quand François Chauveau l'a discrètement évoqué par la présence de quelques embarcations à l'arrière-plan, Hubert-François Gravelot, pour l'édition de 1768 a situé l'action près des murailles de la ville [fig. 6]. La mort du roi Mithridate est connue et nous savons que ce héros racinien fait exception en recourant à deux moyens pour se donner la mort : il prend d'abord du poison, avant de se frapper d'un coup d'épée. Ces faits sont connus par le récit d'Arbate, mais le roi, après avoir agi en coulisses, vient mourir sur la scène et se présenter agonisant à Monime et Xipharès. Les illustrateurs se sont intéressés à la dernière scène de la pièce et ils ont fait le choix de représenter une scène dialoguée plutôt que le récit d'Arbate. La mort du souverain est lente car le poison est inefficace. Racine s'est en effet conformé à l'Histoire puisque Mithridate VI, roi du Pont, s'était immunisé en ingérant d'infimes doses de poison :

8. La Thébaïde, V, 3, v. 1375-1378. 
Pour éviter l'affront de tomber dans leurs mains.

D'abord il a tenté les atteintes mortelles

Des poisons que lui-même a crus les plus fidèles ;

Il les a trouvés tous sans force et sans vertu.

Vain secours, a-t-il dit, que j'ai trop combattu !

Contre tous les poisons soigneux de me défendre,

J'ai perdu tout le fruit que j'en pouvais attendre.

[...]

Aussitôt dans son sein il plonge son épée.

Mais la mort fuit encor sa grande âme trompée?

La vignette de Gravelot [fig. 6] illustre les derniers instants de la vie du roi, sans donner d'indices sur l'acte de suicide. Les armes du premier plan font office de trophée et le roi du Pont porte son épée au côté, rangée dans le fourreau. L'imminence de la mort est cependant sensible, ce qu'indique la posture de Mithridate affaibli, en partie soutenu par ses hommes. Le drame est également contenu dans l'attitude de Monime qui, en pleurs, essuie ses larmes à l'aide de son manteau. Bien qu'elle se tienne légèrement en retrait, son rôle dans l'estampe est essentiel puisqu'elle porte les émotions et se trouve offerte aux regards du spectateur. Son attitude est plutôt convaincante et empreinte d'une certaine grâce. Les soldats sont massés sur les créneaux des murailles ou occupent l'arrière-plan : peu visibles derrière les personnages principaux, leur présence est matérialisée par les lances qui rythment verticalement l'espace.

Mais avant cette scène finale, un autre personnage de cette même tragédie fut près de mourir et prêt à mourir : il s'agit de Monime. La jeune femme a signifié à Phœdime son envie de périr $(\mathrm{V}, 1)$; le poison que le roi lui fait parvenir dans la scène suivante l'incite à agir sur-le-champ. Cependant, celui-là même qui fit porter le poison en ordonne le retrait par son confident au moment où Monime s'apprête à boire. Arbate intime son ordre avec une vivacité extrême, il lui faut arrêter le geste que Monime s'apprête à faire; et le verbe est repris de manière anaphorique. Pour cela il n'use que de l'impératif et, comme l'indique la didascalie, accompagne ses paroles d'un geste définitif. Il lui faut être persuasif car Phœdime avait déjà essayé de retenir sa maîtresse au moment où Arcas avait apporté la coupe. Le rythme du discours est amplement souligné et l'emploi du verbe courir contribue à la dynamique de la scène :

Arbate

Arrêtez! arrêtez!

Arcas

Que faites-vous, Arbate?

Arbate

Arrêtez ! j'accomplis l'ordre de Mithridate.

Monime

9. Mithridate, V, 4, v. 1570-1576 et 1603-1604. 
Ah ! laissez-moi...

Arbate, jetant le poison

Cessez, vous dis-je, et laissez-moi

Madame, exécuter les volontés du roi.

Vivez. Et vous, Arcas, du succès de mon zèle,

Courez à Mithridate apprendre la nouvelle ${ }^{10}$.

Jacques De Sève a restitué dans sa vignette le rythme soutenu du dialogue par une gestuelle expressive qui se substitue aux paroles vives avec efficacité [fig. 5]. Dans le débat de l'ut pictura poesis, les artistes savaient que le langage du silence était celui de leur art. Il n'est pas question pour eux de renforcer le sens d'une phrase par un geste, mais de donner au corps les attitudes et expressions qui conviennent. Arbate fond littéralement sur Monime et arrête son geste avec fermeté. Il arrive au moment où la coupe allait être portée jusqu'aux lèvres. La résignation se lit sur le visage de Monime et l'intervention salvatrice d'Arbate reste invisible pour les femmes qui entourent de la jeune femme. Leurs attitudes indiquent que la mort est assurée. Phœdime refuse de voir l'insoutenable et le dessinateur n'a pas hésité à ne montrer que l'arrière de la tête de la confidente, explicitant cette attitude par le mouvement du bras : elle est presque absente de la scène. Arbate met autant de zèle à la Monime en vie qu'elle en avait mis à tenter de se suicider. Dans la scène suivante il justifie son intervention et narre la fin toute proche de Mithridate que le dessinateur a représentée à l'arrière-plan de la vignette. La composition de Jacques De Sève illustre l'action d'Arbate et, dans cette salle de palais largement ouverte sur l'extérieur, montre Mithridate porté par des soldats tandis que Xipharès se cache la tête dans les mains en signe de douleur. Arbate a quitté le cortège pour sauver la reine ; et, dans le même temps qu'il agit et fait sa relation, la procession poursuit sa progression. Les deux actions évoluent à un rythme qui correspond à celui de la tragédie puisque bientôt le roi mourant se présentera devant Monime et Xipharès. Le vignettiste a hiérarchisé les plans en dessinant avec des traits plus fins l'action des personnages, sans que la disposition parût artificielle ou archaïsante. L'ouverture qui suggère cet autre espace est une occasion pour figurer deux instants chronologiquement très proches sans déroger à la règle de l'unité d'action. En effet, même si les principes des arts visuels diffèrent de ceux du théâtre, les peintres et dessinateurs devaient proposer une chronologie cohérente qui permît de comprendre la succession des événements et les inscrive dans une temporalité. Les théories artistiques, et notamment les Conférences de l'Académie royale de Peinture et de Sculpture du XVII ${ }^{e}$ siècle, ont insisté sur cette nécessité. Il fallait proscrire les archaïsmes et montrer que la peinture méritait le statut d'art libéral. Il s'agissait pour les artistes de parvenir à une harmonie, celle qui préside à la représentation, car seul l'accord des diffé-

10. Mithridate, V, 3, v. 1539-1543. 
rentes parties permet l'éloquence. Le choix de l'instant, le décor, la disposition, les attitudes, les étoffes, tout concourt à cette harmonie que cristallisent l'expression des passions et les mouvements des corps.

Le poison, nous l'avons vu, est un moyen privilégié pour les suicides. Il peut cheminer doucement dans les veines ainsi que le rapporte Phèdre avant d'expirer :

J'ai voulu, devant vous exposant mes remords,

Par un chemin plus lent descendre chez les morts.

J'ai pris, j'ai fait couler dans mes brûlantes veines

Un poison que Médée apporta dans Athènes ${ }^{11}$.

Le breuvage de Médée que la fille de Minos a ingéré n'est pas de même nature que celui que Locuste a élaboré. Le meurtre que Néron prépare nécessite en effet une action rapide et violente, et la mort d'un esclave atteste de la fulgurance de la préparation. La fabrique du poison est un art que Jacques De Sève a représenté dans le cul-de-lampe de l'acte IV de Britannicus:

Le poison est tout prêt. La fameuse Locuste

A redoublé pour moi ses soins officieux :

Elle a fait expirer un esclave à mes yeux ;

Et le fer est moins prompt pour trancher une vie

Que le nouveau poison que sa main me confie ${ }^{12}$.

Ce redoutable poison est destiné à Britannicus puisque la fourberie de Néron a fait son œuvre. Le banquet de la réconciliation est fatal au demi-frère empli d'espoir. Le récit de Burrhus, vibrant, narre la soudaineté du trépas avec des mots qui en disent si bien la violence. Il désigne aussi le meurtrier, laisse voir les visages et entendre les cris :

La coupe dans ses mains par Narcisse remplie,

Mais ses lèvres à peine en ont touché les bords...

Le fer ne produit point de si puissants efforts,

Madame : la lumière à ses yeux est ravie,

Il tombe sur son lit sans chaleur et sans vie.

La moitié s'épouvante et sort avec des cris,

Mais ceux qui de la cour ont un plus long usage

Sur les yeux de César composent leur visage ${ }^{13}$.

Quand certains vignettistes n'ont pas hésité à figurer la mort, d'autres ont minimisé les effets du poison en représentant un homme non pas mort, mais en train de mourir. Ces illustrations s'éloignent quelque peu du récit de Burrhus, comme le montre la vignette de l'édition de 1723 dessinée par Louis

11. Phèdre, V, dernière, v. 1635-1638.

12. Britannicus, IV, 4, v. 1381-1386.

13. Britannicus, V, 5, v. 1618-1625. 
Chéron [fig. 3]. Les bras de Britannicus conservent trop d'énergie et la tête penchée, les yeux fermés traduisent un sentiment de douleur ou d'affliction. Même la fausse surprise de Narcisse - pourtant bien exprimée - ne parvient pas à convaincre le spectateur que Britannicus est déjà mort ${ }^{14}$. On pourra apprécier la dispositio de la scène qui met en valeur l'empereur Néron placé sur un plan plus élevé et installé sous un dais imposant. Il détourne le regard pour ne pas voir ce qui se déroule au premier plan de la composition. Les dimensions de la table insistent sur la distance qui sépare les demi-frères et rappellent combien la réconciliation annoncée n'était qu'un leurre. Enfin, il convient de s'attarder sur la belle vignette de Jacques De Sève [fig. 4]. Le funeste banquet est mis en scène dans un décor soigné avec une perspective sur un jardin. L'escalier, les colonnes géminées, le haut dressoir, le mobilier, l'orfèvrerie et les mets sur la table traduisent un luxe certain. Les lits de banquets de Néron et de Britannicus sont proches, ce qui accroît certainement la jubilation de l'empereur. Pour le spectateur, le visage de Britannicus est à peine visible; seul Néron peut le contempler. Le dessinateur a insisté sur l'expressivité d'un corps renversé aux membres inertes, tandis que la coupe bascule et laisse échapper le poison. La jambe droite encore pliée propose une modulation qui met en valeur le corps terrassé et montre peut-être un vain effort de résistance. Le drame est tout entier contenu dans cette posture qui exprime la fulgurance. Nous avons, pour le combat d'Étéocle et de Polynice, souligné l'importance des personnages secondaires porteurs d'expressions variées. Dans cette vignette, ils se trouvent de l'autre côté de la table et font face au spectateur. Le trouble et l'effroi se lisent sur leurs visages et dans leurs gestes, et plusieurs d'entre eux posent leurs yeux sur Néron. Ce dernier, quant à lui, affiche une forme d'indifférence et, tout en admirant son œuvre, semble s'apprêter à boire, non pas à la réconciliation, mais à sa victoire. Enfin, la complicité de Narcisse s'affiche: il est disposé sur le même plan que Néron, tente de dissimuler l'aiguière et, comme l'empereur porte son regard sur le défunt. Britannicus est ainsi encadré de deux traitres : l'innocence feinte de Néron répond à la fausse surprise de Narcisse.

14. Faut-il reconnaittre dans cette attitude une allusion à Tacite qui décrit deux actions différentes du poison? On peut en douter, car c'est bien au cours d'un banquet que le poison violent emporta Britannicus. Tacite, Annales, Paris, Garnier-Flammarion, 1991, établie par Henri de Bornecques : «Un premier poison lui fut donné par ses précepteurs mêmes, mais il ne fit que traverser ses entrailles, qui s'en délivrèrent, soit que le poison fût trop faible, ou qu'on l'eût mitigé, pour qu'il n'agit pas sur-le-champ. [...] Ils lui promirent alors une mort aussi rapide qu'avec le fer, et c'est auprès de la chambre du prince que l'on distille un breuvage composé de poisons d'une violence éprouvée et foudroyant. [...] Une boisson encore inoffensive et goûtée par l'esclave, mais très chaude, est présentée à Britannicus; puis, comme il la repoussait parce que brûlante, on y verse, mêlé à de l'eau froide, le poison, qui circula si rapidement dans tous ses membres qu'il lui enleva à la fois la parole et la vie ", lib. XIII, XV-XVI, p. 346-347. 
Si ces quelques exemples rappellent, de manière bien incomplète, la beauté et la force des vers raciniens, ils montrent aussi combien les tragédies du dramaturge ont inspiré des compositions variées. La peinture veut égaler les qualités narratives de la poésie qui elle, souhaite susciter des images. Ainsi l'hypotypose et l'ekphrasis répondent-elles aux intentions du poète. Pour éviter que l'un des deux arts ne l'emportât dans l'esprit ou dans le cœur des spectateurs, tous se sont employés à retenir l'attention, à plaire, à émouvoir, mais aussi à persuader. 